\title{
Collectivity, Phase Transitions and Exceptional Points in Open Quantum Systems
}

\author{
W.D. Heiss ${ }^{1,2}$, M. MÜller ${ }^{2}$ AND I. RotTer ${ }^{2,3}$ \\ ${ }^{1}$ Department of Physics, University of the Witwatersrand \\ PO Wits 2050, Johannesburg, South Africa \\ 2 Max-Planck-Institut für Physik Komplexer Systeme \\ 01187 Dresden, Germany \\ 3 Technische Universität Dresden, Institut für Theoretische Physik \\ D-01062 Dresden, Germany
}

05.70.Fh, 03.80.+r, 64.60.-i, 02.30.Dk

\begin{abstract}
Phase transitions in open quantum systems, which are associated with the formation of collective states of a large width and of trapped states with rather small widths, are related to exceptional points of the Hamiltonian. Exceptional points are the singularities of the spectrum and eigenfunctions, when they are considered as functions of a coupling parameter. In the present paper this parameter is the coupling strength to the continuum. It is shown that the positions of the exceptional points (their accumulation point in the thermodynamical limit) depend on the particular type and energy dependence of the coupling to the continuum in the same way as the transition point of the corresponding phase transition.
\end{abstract}




\section{Introduction}

Recently, mechanisms of restructuring of quantum systems are discussed with renewed interest [1] - [7]. Conditions for the formation of collective states on the one hand and of quantum chaos on the other are being studied. Among the oldest examples of the former is the schematic model explaining in simple terms the origin of giant dipole resonances in nuclei [8]. The Hamiltonian is of the form $H=H_{0}+H_{1}=H_{0}+D D^{T}$ where $H_{0}$ is the Hamiltonian of the unperturbed system and $D D^{T}$ describes the factorized dipole-dipole residual interaction. The rank of $H_{0}$ is $N$ where $N$ is the number of unperturbed states considered in a certain energy interval, while the rank of $D D^{T}$ is 1 . If the modulus of the average matrix elements of the vector $D$ is sufficiently large $\left(\bar{D} \gg d_{0}\right.$, where $\bar{D}$ is the average matrix element of $D$ and $d_{0}$ is the mean level distance of the eigenvalues of $H_{0}$ ), one of the eigenstates of $H$ is shifted considerably in energy. Since its eigenvector has contributions from almost all basis states, it is a collective state. This type of collectivity is called internal collectivity 4 .

Besides the internal collectivity, there exists the so-called external collectivity of resonance states [1]. It appears at high level density since the discrete states are in general embedded in the continuum and coupled to each other via the continuum. The Hamiltonian of the open quantum system is, to a good approximation, given by $H_{0}-i V V^{T}$ where $V V^{T}$ contains the coupling matrix elements $V_{j}^{c}$ between the discrete states $j$ and the decay channels $c$. The rank of $V V^{T}$ is equal to the number $K$ of open decay channels. It is usually much smaller than the rank $N$ of $H_{0}$. If the matrix elements of $V V^{T}$ are sufficiently large $\left(\bar{V} \gg d_{0}\right), K$ collective states are formed. They are distinguished from the $N-K$ non-collective states by their large widths or short lifetimes [1] - [7]. The wavefunctions of these $K$ collective resonance states are again characterized by a large number of components of the basis states.

There is a basic difference between internal and external collectivity. Internal collectivity deals with a self-adjoint Hamiltonian, where the coupling parameter is real, while external collectivity is described by a purely imaginary coupling strength. The particular type of collectivity is therefore expressed by a real spectrum in the first case, whereas in the second case the 
dissipative character of the open system is reflected by imaginary parts of the eigenenergies, the physical widths. Formally, the formation of a collective state is, however, the same in both cases. It is expressed by the mixing of the eigenfunctions of the full Hamiltonian with respect to the eigenfunctions of $H_{0}$.

The case $\operatorname{rank}\left(H_{0}\right)=\operatorname{rank}\left(H_{1}\right)$ is not necessarily connected with the appearance of collective states. It has been widely discussed in the literature [9]. Conditions have been given for the occurrence of chaotic behaviour and of some sort of phase transitions $[10$. The mechanism of such restructuring and possible chaotic behaviour in the transitional region $\left(\Lambda \sim \Lambda_{c}\right)$ have been explained in terms of the exceptional points (EPs) of the problem $H_{0}+\Lambda H_{1}$.

The EPs are the only singularities in the complex $\Lambda$-plane of the eigenvalues $E_{k}(\Lambda)$. Their positions are fixed by the choice of $H_{0}$ and $H_{1}$ only. As a consequence, the distribution of the EPs is characteristic for any particular Hamiltonian of the form $H_{0}+\Lambda H_{1}$, once $H_{0}$ and $H_{1}$ are given.

It is therefore natural to discuss the EPs as they determine important properties of the spectrum such as the statistical properties, the type and locations of avoided level crossings, the softness of the spectrum and the ranges of $\Lambda$-values, where special features of the spectrum occur. In particular, the distribution and frequency of the EPs give indications about transitional regions and possible occurrence of chaotic behaviour.

The studies of the EPs have, in the papers quoted, been dealing with closed systems, where the influence of decay channels in the continuum has not been considered, that is $H$ is selfadjoint and the eigenstates are discrete. As stated above we are often faced with dissipative systems, where the coupling to the continuum and decay channels is expressed by complex eigenenergies which give rise to finite life times of resonance states. The complex eigenvalues $\mathcal{E}_{k}=E_{k}-i / 2 \Gamma_{k}$ are interpreted as resonance states at the energies $E_{k}$ with the decay widths $\Gamma_{k}$ which are inverse life times.

While there is a great mathematical similarity in the treatment of the respective self-adjoint and dissipative Hamiltonians, the physical findings deserve particular attention. Of special interest will be the circumstances, 
under which the formation of collective states can be understood as a phase transition. We generalize the results obtained in [10] to open quantum mechanical systems and expand the investigations of [7] by taking into account the effects and properties of the EPs. In all cases, we will restrict ourselves to the one-channel case in which the phase transition as well as the collectivity of one of the states are well pronounced.

Generally, a phase transition is a substantial restructuring of the system taking place at a finite (critical) value $\Lambda_{c}$ of a certain control parameter $\Lambda$. This restructuring is a collective phenomenon, ranging over all scales inherent in the system. The nature of the reorganization process is characterized by the behaviour of an order parameter being a function of $\Lambda$. In our case of an open quantum system the order parameter is $\Gamma_{0} / N$ where $\Gamma_{0}$ is the width of the collective mode (in the one channel case) and $N$ is the total number of states. Its first derivative with respect to $\Lambda$ shows a finite discontinuity at the critical value $\Lambda_{c}$ corresponding to a second-order phase transition. All characteristic features of the phase transition emerge already at finite values of $N$ despite the fact that the strict thermodynamical definition is possible only for $N \rightarrow \infty$. Precise conditions for its occurence are derived in [7].

The phase transition is connected with the appearance of a state whose external collectivity is of a global nature. Its wave function carries contributions from all eigenstates of $H_{0}$. This global collectivity must be distinguished from the local (external) collectivity which appears when the resonance states are coupled strongly to the continuum but the conditions for a phase transition are not fulfilled. The wave function of this collective state carries contributions only from a restricted number of basis states, whose unperturbed energies are overlapped by the width of the collective state [7].

In Section 2 the basics of the EPs and specific features relating to open quantum systems are reviewed. A simple example using two resonances coupled to one decay channel illustrates the connection between the main results known from the study of open quantum mechanical systems [3] and the aspect of the EPs. In Section 3 the restructuring of the system is put into context with several systems of a different nature. As a beginning, the simplest case of a picket fence model is considered. Characteristic patterns of the EPs relating to phase transitions are established. Next, the influence of random 
perturbations within the system is investigated. Finally we discuss the effect of a more general level density distribution of the unperturbed levels and that of the coupling vector $V$. A short summary and outlook is given in Section 4 .

\section{Exceptional points and features of open quantum systems}

The $N \times N$ matrix problem of the form $H_{0}+\Lambda H_{1}$ has eigenvalues $E_{k}(\Lambda), k=$ $1, \ldots, N$ which are obtained by the secular equation

$$
\operatorname{det}\left(H_{0}+\Lambda H_{1}-E\right)=0 \text {. }
$$

We here assume that $H_{0}$ and $H_{1}$ are real and symmetric. The EPs [9] are characterized by the coalescence of any two pairs of eigenvalues. They are fixed by a particular choice of $H_{0}$ and $H_{1}$. If we exclude a genuine degeneracy of eigenvalues for real values $\Lambda$, such coalescence will happen only for complex values of the coupling parameter and hence for complex eigenvalues. Accordingly, the EPs are determined by the simultaneous solutions of Eq.(11) and of

$$
\frac{\mathrm{d}}{\mathrm{d} E} \operatorname{det}\left(H_{0}+\Lambda H_{1}-E\right)=0
$$

where the second equation ensures that two eigenvalues coincide. Eqs.(1) and (2) are polynomials in $E$ and $\Lambda$ of order $N$ and $N-1$, respectively. They can be combined into a single polynomial in $\Lambda$ of order $N(N-1)$, the resultant of Eq.(I), by eliminating the variable $E$. The resultant has real coefficients, hence the EPs occur in $N(N-1) / 2$ complex conjugate pairs. They are the only singularitites which the eigenvalues $E_{k}(\Lambda)$ can have as functions of $\Lambda$. In fact, they are the square root branch points of one analytic function which has $N$ Riemann sheets, where the values on each sheet are just the real eigenvalues $E_{k}(\Lambda)$ for real values $\Lambda$. All $N$ eigenvalues are therefore analytically connected with each other via the square root branch points in the complex $\Lambda$-plane.

The behaviour of the eigenfunctions when continued to the EPs deserves special interest. Since they are lying in the complex $\Lambda$-plane, the operator 
$H_{0}+\Lambda H_{1}$ is no longer self-adjoint at $\Lambda=\Lambda_{\mathrm{EP}}$ with $\Lambda_{\mathrm{EP}}$ denoting a (complex) EP. Therefore we cannot expect two linearly independent eigenvectors even though two (complex) eigenvalues $E_{i}$ and $E_{k}$ coalesce. The eigenbasis of $H_{0}+$ $\Lambda_{\mathrm{EP}} H_{1}$ is no longer orthogonal as it is the case for selfadjoint Hamiltonians. In fact, in contrast to the self-adjoint case, where a twofold degeneracy always implies a two-dimensional eigenspace, an EP is characterized by the fact, that the rank of the associated matrix $H_{0}+\Lambda_{\mathrm{EP}} H_{1}$ drops by one. In other words, there is no two-dimensional subspace associated with the coalescence of two eigenvalues. We rather encounter a confluence of the two eigenvectors $\psi_{l}(\Lambda)$ and $\psi_{k}(\Lambda)$ at $\Lambda=\Lambda_{\mathrm{EP}}$. Moreover, for a general complex value $\Lambda$ not coinciding with an EP, we may choose a biorthogonal system such that

$$
\left\langle\tilde{\psi}_{l}(\Lambda) \mid \psi_{k}(\Lambda)\right\rangle=\delta_{l k},
$$

where $\tilde{\psi}_{i}(\Lambda)$ and $\psi_{i}(\Lambda)$ are the left and the right eigenvectors, respectively. We note, that the transformation $O$ diagonalizing a symmetric complex Hamiltonian $H$ is complex orthogonal $\left(O O^{T}=O^{T} O=1\right)$ but not unitary. Therefore, the scalar product of the two left or right eigenvectors obeys $\left\langle\tilde{\psi}_{k}(\Lambda) \mid \tilde{\psi}_{k}(\Lambda)\right\rangle=\left\langle\psi_{k}(\Lambda) \mid \psi_{k}(\Lambda)\right\rangle \geq 1$ for any $k$. A problem occurs when the normalization condition given by Eq.(3) is considered for $\Lambda \rightarrow \Lambda_{\mathrm{EP}}$ : with $E_{l}\left(\Lambda_{\mathrm{EP}}\right)=E_{k}\left(\Lambda_{\mathrm{EP}}\right)$ it is $\psi_{l}\left(\Lambda_{\mathrm{EP}}\right)=\psi_{k}\left(\Lambda_{\mathrm{EP}}\right)$. This means, in view of Eq.(3), that $\psi_{k}\left(\Lambda_{\mathrm{EP}}\right)$ cannot be normalized at $\Lambda=\Lambda_{\mathrm{EP}}$, since now the orthogonality conflicts with the normalization requirement. Usually, Eq.(3) is globally enforced; as a consequence, the two states $\psi_{l}(\Lambda)$ and $\psi_{k}(\Lambda)$ not only coincide for $\Lambda \rightarrow \Lambda_{\mathrm{EP}}$ but they blow up, that is $\lim _{\Lambda \rightarrow \Lambda_{\mathrm{EP}}}\left\langle\psi_{k}(\Lambda) \mid \psi_{k}(\Lambda)\right\rangle \rightarrow \infty$ [3].

The physical significance of the EPs lies in their relation to avoided level crossing. In particular, in a number of examples it has been demonstrated that in the region of the real values of $\Lambda$, where a high density of EPs occurs, the typical statistical characteristics of the spectrum ascribed to quantum chaos prevail [9, 11].

Turning to open quantum systems

$$
H=H_{0}-i \Lambda V V^{\dagger}
$$

we are faced with a Hamiltonian which has in general complex eigenvalues $\mathcal{E}_{k}=E_{k}-i / 2 \Gamma_{k}$ as $\Lambda$ is complex,

$$
\Lambda=\lambda e^{i \varphi}
$$


(The factor $i$ in front of the coupling term $\Lambda$ in Eq. (4) is used traditionally in this context). The question then arises, whether and under which conditions two (or more) eigenvalues coincide in the complex energy plane and how such a crossing depends on the coupling strength $\Lambda$. The answer to these questions depends on the manner by which the EPs are fixed by the operators $H_{0}$ and $V$.

For illustration let us consider the simple example of two resonances, which are coupled to one open decay channel. The Hamiltonian matrix for this system can be written in the eigenbasis of $H_{0}$ as

$$
\begin{aligned}
H & =\left(\begin{array}{cc}
\epsilon_{1} & 0 \\
0 & \epsilon_{2}
\end{array}\right)-i \Lambda\left(\begin{array}{cc}
\cos ^{2} \omega & \cos \omega \sin \omega \\
\cos \omega \sin \omega & \sin ^{2} \omega
\end{array}\right) \\
& \equiv H_{0}-i \Lambda V V^{\dagger},
\end{aligned}
$$

where we use $H_{1}=V V^{\dagger}$. The relative coupling strength of the two resonance states to the continuum is determined by the angle $\omega$ of the vector $V=$ $(\cos \omega, \sin \omega)$. The simplicity of the model provides an analytic expression for the two EPs, viz.

$$
\Lambda_{\mathrm{EP}}=i\left(\epsilon_{2}-\epsilon_{1}\right) e^{ \pm 2 i \omega}
$$

which are the zeros of the square root in the expression for the eigenenergies, which read

$$
\mathcal{E}_{1,2}=\frac{\epsilon_{1}+\epsilon_{2}-i \Lambda}{2} \pm \frac{1}{2} \sqrt{\left(\epsilon_{1}-\epsilon_{2}\right)^{2}-2 i \Lambda\left(\epsilon_{1}-\epsilon_{2}\right) \cos 2 \omega+(i \Lambda)^{2}} .
$$

The example nicely demonstrates that the EPs depend only on $H_{0}$ and $H_{1}$. Under variation of the angle $\omega$ the EPs are moving on a circle with radius $\epsilon_{2}-\epsilon_{1}$ in the complex $\Lambda$-plane. What determines the EPs are the energies of the unperturbed states (that is $H_{0}$ ) and their relative coupling (that is $H_{1}$ ), and not the phase $\varphi$ of the coupling $\Lambda=\lambda e^{i \varphi}$.

Yet, the complex eigenvalues $\mathcal{E}_{1,2}$ obviously do depend on $\Lambda$, that is on its modulus $\lambda$ and on the angle $\varphi$. Variation of $\lambda$ invokes trajectories in the complex energy plane. The contours of the trajectories depend therefore on both, the phase $\varphi$ and the relative coupling controlled by $\omega$. For $\omega=45^{0}$ and $\varphi=\varphi_{\mathrm{EP}}=0^{0}$ the two trajectories will cross one EP at $\lambda=\lambda_{\mathrm{EP}}=\epsilon_{2}-\epsilon_{1}$. At this critical value of the coupling strength the two eigenvalues coalesce. The 
dimension of the eigenspace of the Hamiltonian is reduced to one including the consequences for the eigenvectors mentioned above. We stress, however, that the crossing of trajectories should not be confused with the familiar degeneracy for self-adjoint Hamiltonians.

For $\varphi \neq \varphi_{\mathrm{EP}}$ the two trajectories repel each other within a certain finite distance in the complex plane [3]. This is a generalization of avoided level crossing for real eigenvalues of a hermitian Hamilton operator. Only if $\varphi$ is properly tuned to the value of $\omega$ can the two energy trajectories genuinely cross each other. A nice illustration of this simple example for different values of $\omega$ and $\varphi$ can be found in [6].

What becomes obvious in the $2 \times 2$ matrix model can be generalized to an arbitrary $N \times N$ situation, i.e. $N$ resonance states coupled to one common decay channel. With increasing $\lambda$ one eigenvalue trajectory always drifts further into the complex plane, while the others are bending back towards the real axis after they have repelled with the collective state whose width always increases. This happens irrespective of $\varphi$. Physically it means that one of the resonance states takes almost all of the transition strength by trapping the others which then become long-lived [3, 6].

This is understood by the rank one of the coupling matrix. At large values of $\lambda$ the first part $H_{0}$ of the Hamiltonian is a small perturbation. Therefore, the total matrix is essentially turned into an operator of rank 1 . In other words, there is only one nonzero eigenvalue, and the widths of the zero eigenvalues have to vanish. In general, with $N$ resonance states and $K<N$ open decay channels, there appear $K$ fast decaying states and $N-K$ states which are virtually stable. Various examples of the many channel - many resonance case can be found in [1] - [7].

The low rank of $H_{1}$ has a drastic effect on the total number of EPs. If $K$ is the rank of $H_{1}$, the number of EPs is $K(2 N-K-1)$. The important point is the linear behaviour in $N$; only when $K$ attains the order of magnitude of $N$ is the quadratic behaviour retrieved. This finding is significant in that it indicates that chaotic behaviour cannot be generated by a low rank of $H_{1}$, there is simply an insufficient number of avoided level crossings. This is in line with an analysis of the level statistics of a Poisson ensemble coupled to 
a continuum by a Gaussian coupling vector [2, 5], where, for large coupling strength, chaotic features of the system are the more pronounced the larger the number $K$ of decay channels.

\section{Results}

We turn our attention to the critical region, where a restructuring of the system occurs. We address the relationship between the potential occurrence of a phase transition which is associated with the formation of a globally collectice state and the distribution of the EPs. Since the essential properties of the Hamiltonian must be reflected in the distribution of the EPs, we expect this relationship to exist.

\subsection{Simple example: picket fence}

We consider the simple model with

$$
H_{0}=\left(\begin{array}{cccc}
-\frac{N-1}{2} & 0 & \cdots & 0 \\
0 & -\frac{N-3}{2} & \cdots & 0 \\
\vdots & & \ddots & \vdots \\
0 & \cdots & 0 & \frac{N-1}{2}
\end{array}\right)
$$

and the $N \times N$ matrix of rank one

$$
V V^{\dagger}=\left(\begin{array}{ccc}
1 & \cdots & 1 \\
\vdots & & \vdots \\
1 & \cdots & 1
\end{array}\right)
$$

The Hamiltonian describes a picket fence spectrum, where all states are coupled equally to one decay channel. Such a system shows a phase transition at $\Lambda=1 / \pi[7]$. Results for finite $N$ can be obtained easily by numerical means. In the limit $N \rightarrow \infty$ the zeros of $\operatorname{det}\left(H_{0}-i \Lambda V V^{\dagger}-\mathcal{E}\right)$ and $\sin (\pi \mathcal{E})-i \Lambda \cos (\pi \mathcal{E})$ coincide [7]. One finds an accumulation point of the EPs, which emerges in the limit and is found from the zeros of the resultant, which is obtained by 
eliminating the variable $\mathcal{E}$ from the set

$$
\begin{aligned}
& \sin (\pi \mathcal{E})+i \pi \Lambda \cos (\pi \mathcal{E})=0 \\
& \cos (\pi \mathcal{E})-i \pi \Lambda \sin (\pi \mathcal{E})=0
\end{aligned}
$$

These two equations are equivalent to Eqs. (11), (2). Their simultaneous solutions, the zeros of the resultant (denoted by Rsl), are given by

$$
\operatorname{Rsl}(\lambda)=\sqrt{1+(i \pi \Lambda)^{2}}
$$

Obviously, this is no longer a polynomial. The important point of our finding is that all roots which occur in the complex $\Lambda$-plane for finite $N$ converge to $\Lambda=\lambda= \pm 1 / \pi$ in the limit $N \rightarrow \infty$. Negative values of $\lambda$ would lead to negative decay widths which have no physical meaning. In the following, we restrict ourselves to positive $\lambda$ and $0^{0} \leq \varphi \leq 90^{\circ}$, since all relations are symmetric with respect to the replacement $\Lambda \rightarrow-\Lambda$ and $\Lambda \rightarrow \Lambda^{*}$. Note also that the limit point, being a point of accumulation, is no longer a square root branch point for the energy spectrum but rather a logarithmic branch point. In fact, the analytic behaviour of the (infinitely many) energy levels $\mathcal{E}_{k}(\Lambda)$ in the vicinity of $\Lambda=1 / \pi$ is found by solving the secular equation explicitly for $\mathcal{E}$. The expression reads

$$
\mathcal{E}(\Lambda)=\frac{1}{\pi} \operatorname{atan}(-i \Lambda \pi)=\frac{i}{2 \pi} \ln \frac{1-\Lambda \pi}{1+\Lambda \pi}
$$

which clearly reveals the logarithmic branch points at $\Lambda=1 / \pi$. Moreover, for $\lambda \pi<1$ we read off

$$
\mathcal{E}_{k}=k-i \Lambda+O(2)
$$

while for $\lambda \pi>1$

$$
\mathcal{E}_{k}=k+\frac{1}{2}+\frac{i}{2 \pi} \ln \left|\frac{1-\Lambda \pi}{1+\Lambda \pi}\right|=k+\frac{1}{2}-\frac{i}{\pi^{2} \Lambda}+O\left(\Lambda^{-2}\right)
$$

with $k$ integer. In both cases, the remaining terms, denoted by $O$, are purely imaginary if $\varphi=0^{0}$. These results were obtained in [0].

For finite $N$ the resultant relating to the Hamiltonian $H_{0}-i \Lambda V V^{\dagger}$ becomes a polynomial of order $N-1$ in $\Lambda^{2}$. The complex roots which are the 
EPs are therefore not only symmetric with respect to the real axis but a solution $\Lambda_{\mathrm{EP}}$ implies also the solution $-\Lambda_{\mathrm{EP}}$. As a consequence, a solution has to occur on the real $\Lambda$-axis for $N$ even. This is a non-generic feature of the present model. For odd values of $N$ and $\varphi=0^{0}$, no eigenvalue is crossing an EP under variation of $\lambda$. We use such cases in our numerical demonstrations.

To illustrate how the $\mathcal{E}$ undergo level repulsions and how the repulsions are related to the crossing points $\mathcal{E}\left(\Lambda_{E P}\right)$, we display in Fig.1 the crossing points and the eigenvalue trajectories for different values of $N$ and $\varphi=0^{0}$. Since the spectrum is symmetric with respect to positive and negative energies, only the positive part near to the centre is drawn. For $\Lambda=0$ all trajectories begin at the unperturbed energies $\epsilon_{k}$, move into the complex plane and then turn back $\left(\epsilon_{k} \neq 0\right)$ towards the real axis again. For large $N$ the corresponding values are given by Eq. (16) (which is valid for $N \rightarrow \infty$ ). With increasing $N$ the imaginary parts of the turning and the crossing points increase, while they move nearer to each other. The trajectory of the collective state $\left(\epsilon_{k}=0\right)$ is moving on the imaginary axis towards larger imaginary values implying an increasing width $\Gamma$. We interpret the turning points as level repulsions of the collective state with the other levels. The descending slope of the envelope of the turning points and the crossing points is due to the finite size of the spectrum.

In Fig. 2 we display the EPs in the $\Lambda$-plane for a few values of $N$. The sets of the EPs belonging to the same $N$ are connected by a solid line. The zooming in of the EPs towards the accumulation point $\lambda_{c}=1 / \pi$ for increasing $N$ is clearly discernible and enhanced in the insert of Fig.2. The larger $N$ the larger is the density of the EPs near to the real axis. Additional points come in further away with each additional step of $N$. They are typical edge effects and correspond to the turning points of the energy trajectories at the outer edges of the spectrum. These points which emerge further away from the real axis quickly move in to get near to the others while new further points come up for the following steps of $N$. Eventually, in the limit $N \rightarrow \infty$, all points coalesce at the accumulation point.

In this way, variation of the coupling strength $\Lambda$ invokes a certain trajectory of the system in the complex $\Lambda$-plane. If it hits the accumulation point $\Lambda_{c}$ (or the high-density regions of EPs at finite $N$ ), all complex eigenvalues 
coalesce (nearly coalesce) at their crossing point. This leads to a sharp transition from a regime with $N$ resonance states to a regime with one collective state and $N-1$ trapped states.

We note that at the accumulation point $\Lambda=\Lambda_{c}$, which can occur only in the limit $N \rightarrow \infty$, all infinitely many eigenstates collaps into one. It is the global collective state. It retains its characteristics also for $\lambda>\lambda_{c}$, when the other states (the trapped states) re-emerge. This particular case underlines the crucial connection between the critical point of a phase transition and the EPs.

In the picket-fence model the accumulation point $\Lambda_{c}$ lies on the real axis. If for physical reasons, a value $\varphi \neq 0$ has to be chosen with $H_{0}$ and $H_{1}$ left unchanged, a variation of $\lambda$ will effect a trajectory in the complex $\Lambda$-plane that passes the high-density region (or accumulation point for infinite $N$ ) at a certain distance. This affects the sharpness of the transition between the two regimes. Note that this type of softening of the phase transition is different in nature from the one caused by a finite value of $N$. It persists in the limit $N \rightarrow \infty$. This underlines that $\varphi$ as well as the distribution of the EPs determine, whether there is a phase transition in the strict thermodynamical sense, which is associated with the sudden formation of a globally collective state in the system.

As an aside we realize that the schematic model $\left(\varphi=90^{\circ}\right)$ cannot have any signatures of a sharp phase transition, when the real value of $\lambda$ is varied from zero to large values. The accumulation point is then relatively far away on the imaginary axis of the coupling strength. Results for a variety of angles $\varphi$ but the same $H_{0}$ and $H_{1}$ have been investigated in [7].

Previously, a criterium for a phase transition, viz.

$$
B=\frac{1}{2 N+1} \sum_{k=-N}^{N}\left\langle\psi_{k} \mid \psi_{k}\right\rangle
$$

has been introduced in [7]. Excluding the non-generic case of an accidental crossing of two (or more) eigenvalues at an EP, $B$ is an indicator of the sharpness of the transition. On the one hand, it is the larger the smaller the 
distance between the complex eigenvalues and their crossing points. This distance is determined by the angle $\varphi$. On the other hand, $B \gg 1$ only if all complex eigenvalues reach their minimum distance simultaneously at the same value $\Lambda=\Lambda_{c}$, i.e. if the EPs accumulate. In this case, $B$ shows a pronounced maximum as a function of $\Lambda$ around $\Lambda=\Lambda_{c}$.

In Fig.3 a few cases including the picket fence model are illustrated. It also demonstrates a situation where a local collective state is formed, which means that the phase transition is washed out completely. We return to this latter aspect at the end of Section 3.3.

To summarize the findings of this subsection: the high density of EPs provides the mathematical mechanism for the restructuring of the system under variation of the modulus $\lambda$ of the coupling parameter $\Lambda$ towards larger values. The sharpness of the transition which is evoked by the variation is determined by the distance at which the high density region (accumulation point for infinite $N$ ) of EPs is passed by the corresponding energy trajectories. This distance is determined by the angle $\varphi$.

\subsection{Random change of the unperturbed spectrum}

The essential aspects of our findings remain unchanged if the unperturbed energies in $H_{0}$ and/or the elements of the coupling matrix $V$ deviate from the symmetrical form used in the previous Section. We address the question, whether and to what extent such disturbance can change the basic pattern, that is the formation of a region of high density of EPs or even of an accumulation point in the large $N$ limit.

For this purpose, we define random perturbations in $H_{0}$ by $\left(H_{0}\right)_{k, k}=$ $-(N-1-2 k) / 2+r_{k}$ where the $r_{k}$ are random numbers from a uniform distribution in the interval $[-0.1,0.1]$. In Fig.4 we have drawn the EPs for the perturbed and unperturbed picket fence in the complex $\Lambda$-plane for $N=19$. As in Fig.2, the unperturbed EPs are connected by a solid line. The perturbed EPs do no longer lie on the smooth curve but they are scattered around it. 
The quantity $B$ of Eq. (17) indicates the accumulative behaviour of the EPs. It is illustrated in Fig.3 for the disturbed picket fence model as a function of $\lambda$ for two different values of $N$ using $\varphi=0^{0}$. The excess beyond unity of $B$ around $\lambda=1 / \pi$ increases with increasing $N$. We interpret this result as a strong indication for the disturbances to be washed out, the more so the larger $N$. In other words, for an increasing number of states, the average distribution of the EPs is zooming in to $\Lambda_{c}=\lambda_{c}=1 / \pi$. Hence, the EPs of the irregular system accumulate on the average.

Furthermore, the precice form of the distribution from which the random changes in $H_{0}$ and/or $H_{1}$ are drawn is immaterial. In particular, choosing the eigenvalues of $H_{0}$ from a Wigner (GOE) or a Poissonian distribution, does not alter our conclusions. The existence and the position of the accumulation point remains unaffected.

\subsection{Level density dependence}

In real physical systems, the level density, the number and the coupling strength of the decay channels are in general energy dependent. The former is usually a monotonically increasing function, whereas for instance in nuclear physics the continuum coupling strength decreases. This energy dependence will influence the distribution of the EPs.

In [7] it has been shown that it needs a proper tuning between the density dependence on the one hand and the coupling dependence on the other in order to guarantee a phase transition at a finite value of the coupling strength. Given the energy dependence of the level density $\rho(E)$, a phase transition occurs at finite coupling strength, if and only if the energy dependence of the coupling vector is given by the inverse function $\rho(E)^{-1}$. If the system obeys this condition on the average, a phase transition still occurs. The critical point may be shifted with respect to the value of the ideal picket fence model. We talk about 'overcompensation' when the energy dependence of the coupling vector decreases (increases) at a lesser (faster) rate than that of the inverse behaviour of the level density. In this case, numerical results [0] led to the conjecture that the critical point is shifted to zero, i.e. $\lambda_{c} \rightarrow 0$ if $N \rightarrow \infty$. In the opposite case, which we denote as 'undercompensation', 
a global collective mode and a global reorganisation of the spectrum as a whole is absent. Now one obtains $\lambda_{c} \rightarrow \infty$ if $N \rightarrow \infty$. The occurence of the broad mode remains then a local phenomenon for all finite values of $\lambda$ [7]. A collapse of the Hilbert space as in the picket-fence model does not occur.

The following substantiates the conjecture about the position of $\lambda_{c}$ in the under- and overcompensated cases. To facilitate the discussion we restrict ourselves to a power behaviour of the energy dependence of the coupling and level density. The energy dependence of the coupling matrix elements $\left|v_{k}\right|^{2}$ is assumed to be of the form $|k|^{r}$. The unperturbed energies (eigenvalues of $\left.H_{0}\right)$ are defined as $\epsilon_{k}=\operatorname{sign}(k)|k|^{t / 2}$. The secular equation Eq. (11) can be written as

$$
\sum_{k=-N / 2}^{N / 2} \frac{\left|v_{k}\right|^{2}}{\mathcal{E}-\epsilon_{k}}=\frac{i}{\Lambda} .
$$

Using (18), we obtain

$$
\sum_{k=-N / 2}^{N / 2} \frac{\left|v_{k}\right|^{2}}{\left(\mathcal{E}-\epsilon_{k}\right)^{2}}=0
$$

which corresponds to Eq. (2)).

The EPs are simultaneous solutions of Eqs.(18) and (19). It is obvious from Eq.(19) that the energy values at the EP cannot be real. In fact, it is known [7], and also discussed in connection with Fig.1, that, for $t=2$ and $r=0$ (picket fence), the imaginary parts of the energies at the EP tend to infinity for $N \rightarrow \infty$. Numerical evidence as well as the following consideration supports the conjecture that this holds for arbitrary values of $r$ and $t$ as long as $t-r>1$. For this purpose we rewrite Eq.(18) for even $N$

$$
2 \mathcal{E} \sum_{k=1}^{N / 2} \frac{k^{r}}{\mathcal{E}^{2}-k^{t}}=\frac{i}{\Lambda} .
$$

In the large $N$ limit we replace the sum in Eq.(20) by an integral and $\mathcal{E}$ by $i z$ and obtain

$$
\begin{aligned}
-2 i z \int_{1}^{\infty} \frac{k^{r} \mathrm{~d} k}{z^{2}+k^{t}} & =\frac{-2 i z}{t-r-1} F_{21}\left(1,1-\frac{1+r}{t}, 2-\frac{1+r}{t} ;-z^{2}\right) \\
& =\frac{i}{\Lambda} .
\end{aligned}
$$


The derivative with respect to $z$ of the right hand side of Eq.(21), which is a linear combination of two Hypergeometric Functions, corresponds to the large $N$ limit of Eq.(19) $(\mathcal{E} \rightarrow i z)$. It is straighforward to show, for instance by graphical means, that this derivative has no zero for finite values of $z$. In fact, the derivative vanishes only for $z \rightarrow \infty$ irrespective of $r$ and $t(t>r+1)$. We exploit this fact in Eq.(18) in that we consider the limit of the left hand side for large imaginary values of $\mathcal{E}=E-i \Gamma / 2$. It is found to be proportional to $\Gamma^{2(1+r) / t} / \Gamma$. This yields the generalised result that the overcompensated case occurs for $2(1+r)>t$, and the compensated and undercompensated cases for $2(1+r)=t$ and $2(1+r)<t$, respectively [7]. In fact, the respective limit of the left hand side of Eq.(21) is infinity, a finite constant and zero for $\Gamma \rightarrow \infty$. Consequently, the corresponding values of $\lambda$ must be zero, finite and infinity, respectively. Since these values constitute simultaneous solutions of Eqs.(18) and (19), they are the values where the EP accumulate. Furthermore it follows, that the accumulation point is on the real axis for the compensated case $(t=2(1+r))$.

To demonstrate further this result, the values of $B$ as a function of $\Lambda=\lambda(\varphi=0)$ are drawn for the compensated case $r=1, t=4$ in Fig.3. One clearly sees that the maximum of $B$ around $\lambda=2 / \pi$ increases with $N$. As explained above, this means that, on the average, the minimum distance between the crossing point and its correponding eigenvalues decreases with increasing $N$. In the limit $N \rightarrow \infty$, all eigenvalues will hit the crossing point. Thus, the compensated system behaves similar to the picket fence. Also in this case, the space of the eigenstates collapses at $\Lambda=\Lambda_{c}=2 / \pi$ into dimension one, and a globally collective state is created. For comparison, the undercompensated case with $r=0$ and $t=4$ is drawn in Fig. 3 as an example for a system which does not undergo a phase transition. As explained above, $\lambda_{c} \rightarrow \infty$ in this case and $B$ has no maximum at a finite value of $\lambda$.

\subsection{Phase behaviour of the wave functions}

In Section 2 some properties of the eigenfunctions have been pointed out, when a non self-adjoint Hamiltonian is considered. We resume the discussion in more detail here with particular emphasis on the phase of the wave functions when an EP is approached by the variation of the coupling pa- 
rameter $\Lambda$. Surrounding one crossing point in the complex energy plane corresponds to a double loop around the EP in the $\Lambda$-plane, Eq. (7), since the EPs are square root branch points, Eq. (8). We address in the following the effect of looping once around an EP in the $\Lambda$-plane. For demonstration we use the simple two dimensional model introduced in Section 2, Eq. (6).

The two eigenfunctions, normalized according to Eq. (3), can be parametrized by the complex angle $\theta$ :

$$
\psi_{1}=\left(\begin{array}{c}
\cos \theta \\
\sin \theta
\end{array}\right), \quad \psi_{2}=\left(\begin{array}{c}
-\sin \theta \\
\cos \theta
\end{array}\right)
$$

where the angle $\theta$ is given by

$$
\tan ^{2} \theta=\frac{\mathcal{E}_{1}-\mathcal{E}_{2}-\left(\epsilon_{1}-\epsilon_{2}\right)+i \Lambda \cos 2 \omega}{\mathcal{E}_{1}-\mathcal{E}_{2}+\left(\epsilon_{1}-\epsilon_{2}\right)-i \Lambda \cos 2 \omega}
$$

The notation has been introduced in Eqs. (6) and (8). From this expression we read off:

(i) at an $\operatorname{EP}\left(\mathcal{E}_{1}=\mathcal{E}_{2}\right)$ we obtain $\tan ^{2} \theta=-1$ which implies $|\cos \theta|=$ $|\sin \theta|=\infty$, that is the components of the wave functions blow up;

(ii) when an EP is surrounded in the $\Lambda$-plane (which amounts to $\mathcal{E}_{1}-\mathcal{E}_{2} \rightarrow$ $\left.\mathcal{E}_{2}-\mathcal{E}_{1}\right)$, the $\tan ^{2} \theta$ is changed into $1 / \tan ^{2} \theta$, which corresponds to the change $\theta \rightarrow \theta+\pi / 2$.

This implies $\psi_{1} \rightarrow \psi_{2}$ and $\psi_{2} \rightarrow-\psi_{1}$.

While it may not be obvious to implement such a contour in the complex $\Lambda$-plane in an actual physical experiment, there could be a possibility to achieve the same effect by a variation of the modulus of $\Lambda$ for different values of the relative coupling given by $\omega$. In fact, using the setting $H_{0}-i \lambda V V^{\dagger}$, then the EP lies for $\omega \gtrsim 45^{0}$ just below and for $\omega \lesssim 45^{0}$ just above the point $\lambda=\epsilon_{2}-\epsilon_{1}$. If we compare the two situations while varying $\lambda$, the two wave functions for $\lambda>\epsilon_{2}-\epsilon_{1}$ differ in the same way as if the EP had been surrounded. 
This can be made explicit by choosing an expression for $\tan \theta$ which is more convenient for this purpose, viz.

$$
\tan \theta=\frac{-i \lambda \sin 2 \omega}{\mathcal{E}_{1}-\mathcal{E}_{2}+\epsilon_{1}-\epsilon_{2}-i \lambda \cos 2 \omega}
$$

The difference between the two values for $\omega$ manifests itself in the difference of the sign of the imaginary part of $\mathcal{E}_{1}-\mathcal{E}_{2}$, since different Riemann sheets have been approached. As a consequence, for large values of $\lambda$ the right hand side of Eq. (24) tends towards $\tan \omega$ for the one case and towards $-\cot \omega=\tan (\pi / 2+\omega)$ for the other.

We suggest that using electro-magnetic resonators may allow control of both, the global coupling $\lambda$ and the relative coupling between two resonances which is given by $\omega$. If it should be possible to bring to interference the wave functions for the two different situations, that is for $\omega \gtrsim 45^{0}$ and for $\omega \lesssim 45^{0}$, the different phases are expected to be observable.

The situation described is reminiscent of Berry's phase [12]. However, we stress that in our discussion we are dealing with non self-adjoint operators, and an EP is therefore not to be confused with Berry's diabolic points. This difference was pointed out in [13]. In fact, a generic EP (i.e. when just two levels coalesce) gives rise to a double pole in the corresponding Green's function or S-matrix. The mechanism for this to occur is again related to the vanishing of the norm of the eigenvectors when $\Lambda \rightarrow \Lambda_{\mathrm{EP}}$. The double pole is an additional signature for an EP. It is different from the simple pole emerging in the Green's function (S-matrix), if a usual single resonance or an incidental degeneracy of two resonances occurs, say, owing to some symmetry. The latter situation is, however, an unlikely event as it needs the tuning of four parameters (six parameters in the case of no time reversal) to achieve it. In contrast, the existence of the EPs is an intrinsic mathematical feature.

\section{Summary and Outlook}

In this paper, we studied the relation between phase transitions, collective states and the distribution of EPs of open quantum systems. Since both the

collectivity and the phase transition are well pronounced in the one-channel 
case, we restricted ourselves to an $N$-dimensional quantum system coupled to one open decay channel. Our results are as follows:

(i) A necessary condition for a phase transition to occur is that the EPs accumulate in the complex $\Lambda$-plane. This result has been shown analytically for the picket-fence model and some generalisations, and is confirmed by numerical results using the quantity $B$. The quantity $B$ is a measure for the average minimum distance of the eigenvalues to its crossing point in the complex energy plane. In the cases considered, $B$ has a pronounced maximum at the critical value $\Lambda_{c}$ at which the phase transition occurs. The increase of $B$ with the number $N$ of states is a further indication that the EPs accumulate in the complex $\Lambda$-plane.

(ii) The choice of the angle $\varphi$ is dictated by the physical situation. Only if it is equal to the phase of $\Lambda_{c}$, the system hits the accumulation point (or goes through the region of high density of EPs), when $\lambda$ is varied. Otherwise, the system may pass the vicinity of the accumulation point (or the region of high density of EPs) and a phase transition in the strict thermodynamical sense does not occur. In all cases considered, we find that $\varphi$ has to be $0^{0}$ for hitting the accumulation point $\Lambda_{c}$ and for a genuine phase transition to emerge.

(iii) If the system hits the accumulation point, the infinite-dimensional space collapses to a one-dimensional function space, since the infinitely many eigenfunctions are identical at $\Lambda=\Lambda_{c}$. This is in contrast to the function space of a usual $N$-fold degeneracy, where $N$ independent eigenfunctions occur. The eigenfunction of the collective state contains contributions of all basis functions of the unperturbed system $H_{0}$. It is a globally collective state which is created by the system as a whole.

(iv) If the system does not hit the accumulation point, the formation of the collective state is not related to a genuine phase transition. This is in particular the case for a system with a purely hermitian coupling matrix $H_{1}$ $\left(\varphi=90^{\circ}\right)$ which creates a state with large internal collectivity.

(v) If the accumulation point is at $\Lambda_{c} \rightarrow \infty$, the formation of the collective state occurs successively and locally. Its wavefunction contains contributions 
only from those resonance states which are overlapped by it.

Exploiting the fact that the crossing points in the complex energy plane are square root branch points, we suggest a possibility for an experimental study of the level repulsion of the complex eigenvalues. For this, the relative coupling strength of the states to the continuum as well as the overall value of their coupling strength should be controllable. A signature of local resonance crossing is given by a particular change of the phase of the complex eigenfunctions. Work towards this aim is in progress.

Acknowledgement: Valuable discussions with T. Gorin, C. Jung, S. Muraviev and G. Soff are gratefully acknowledged. The present investigations are supported by DFG and SMWK.

\section{References}

[1] I. Rotter, Rep. Prog. Phys 54, 635 (1991); V.V. Sokolov and V.G. Zelevinsky, Ann. Phys. (N.Y.) 216, 323 (1992); M. DesouterLecomte, J. Liévin and V. Brems, J. Chem. Phys. 103, 15 (1995)

[2] F.M. Dittes, I. Rotter and T.H. Seligman, Phys. Lett. A158, 14 (1991)

[3] M. Müller, F.-M. Dittes, W. Iskra and I. Rotter, Phys. Rev. E52 5961 (1995)

[4] V.V. Sokolov, I. Rotter, D.V. Savin and M. Müller, Phys. Rev. C56, 1031 and 1044 (1997)

[5] T. Gorin, F.M. Dittes, M. Müller, I. Rotter and T.H. Seligman, Phys. Rev. E56, 2481 (1997)

[6] E. Persson, T. Gorin and I. Rotter, Phys. Rev. E (in press)

[7] C. Jung, M. Müller and I. Rotter, quant-ph/9804020

[8] G. Brown and M. Bolsterli, Phys. Rev. Lett. 3, 472 (1959) 
[9] W.D. Heiss and A.L. Sannino, J. Phys. A23 1167 (1990); W.D. Heiss and A.L. Sannino, Phys. Rev. A43 4159 (1991)

[10] W.D. Heiss, Phys. Rep. 242, 443 (1994)

[11] W.D. Heiss and A.A. Kotzé, Phys. Rev. A44, 2403 (1991); A.A. Kotzé and W.D. Heiss, J. Phys. A: Math Gen, 27, 3059 (1994)

[12] M.V. Berry, Quantum Chaos, ed. by G. Casati (London: Plenum) 1985; Proc. R. Soc. A239, 45 (1983)

[13] E. Hernandez and A. Mondragon, Phys.Lett B326, 1, (1994); A. Mondragon and E. Hernandez, J. Phys. A26, 5595 (1993)

\section{Figure Captions}

\section{Figure 1}

The trajectories of the eigenvalues for $N=15$ and $N=43$ for increasing $\lambda \in[0.001,2]$ in steps of 0.001 . The crossing points are marked by diamonds $(N=15)$ and crosses $(N=43)$.

\section{Figure 2}

The exceptional points in the complex $\Lambda$-plane for $N=15$ (rhombs), $N=19$ (plus signs), $N=27$ (crosses) and $N=43$ (triangles). The inset is a magnification around the accumulation point $\Lambda_{c}$ (black square). The arrows indicate the changes of the EPs with increasing $N$.

\section{Figure 3}

$B$ as a function of $\lambda$ for different systems with $\varphi=0$ : ideal picket fence with $N=101$ (dotted line), randomly perturbed picket fence with $N=101,1001$ 
(solid lines), compensated case with $r=1, t=4$ and $N=101,1001$ (dashed lines), undercompensated case with $r=0, t=4$ and $N=101$ (thick line). The two values $\lambda_{c}=1 / \pi$ and $2 / \pi$, referring to $N \rightarrow \infty$, are indicated by a vertical solid line.

\section{Figure 4}

The EPs for $N=19$ for the ideal picket fence (rhombs) and the randomly perturbed picket fence (plus signs). $\Lambda_{c}$ is denoted by a black square. 


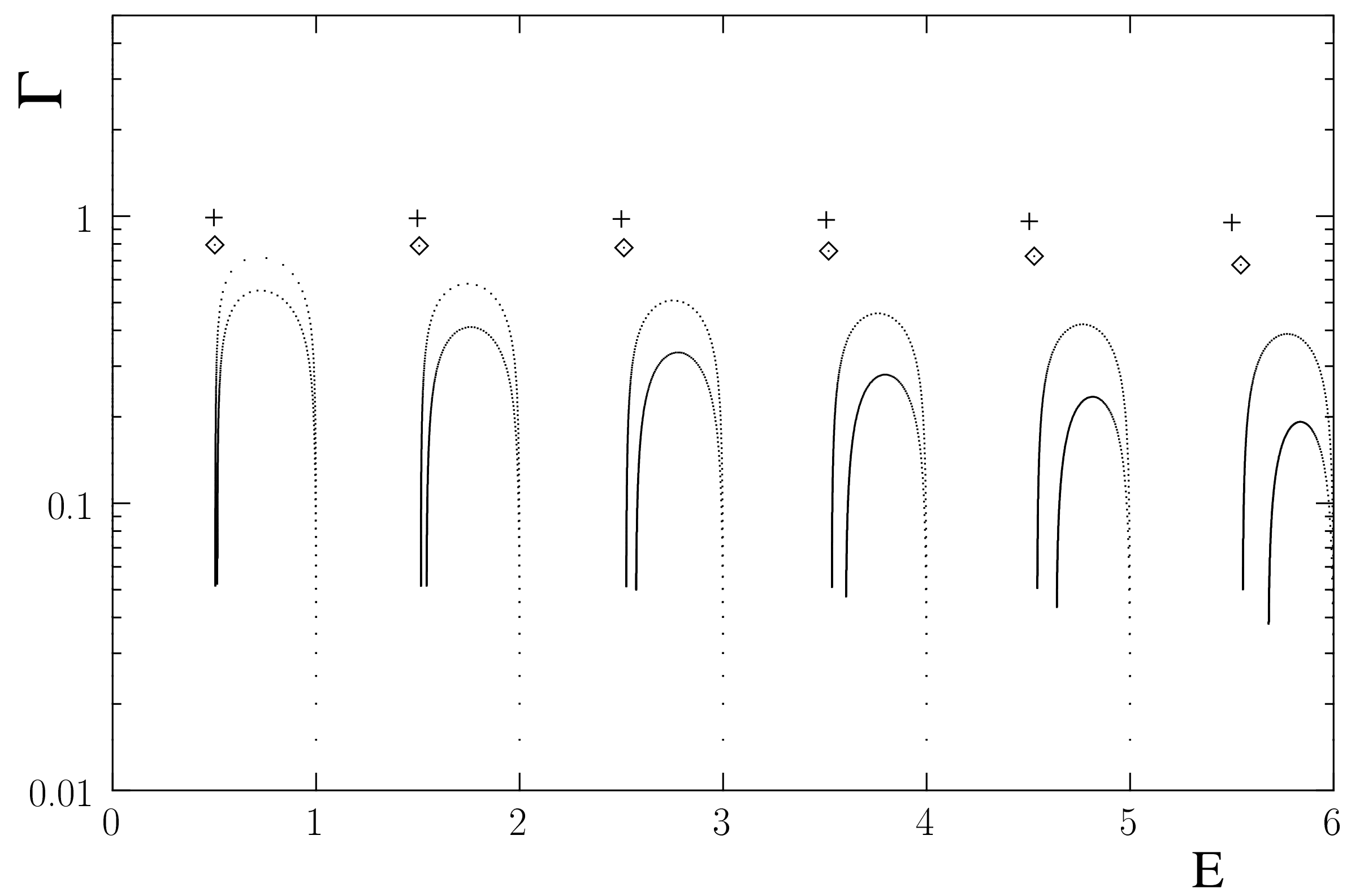

Fig. 1 


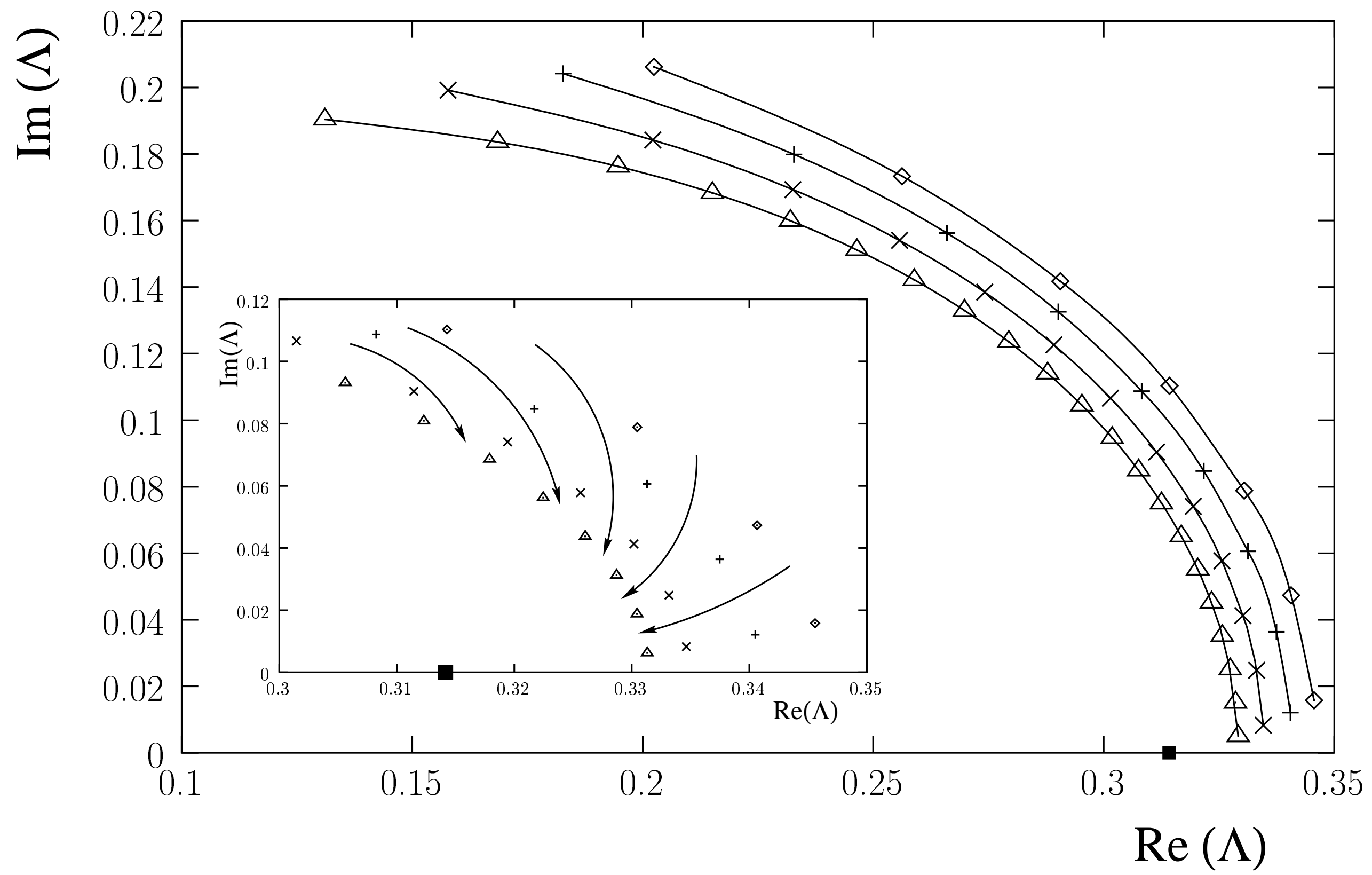

Fig. 2 


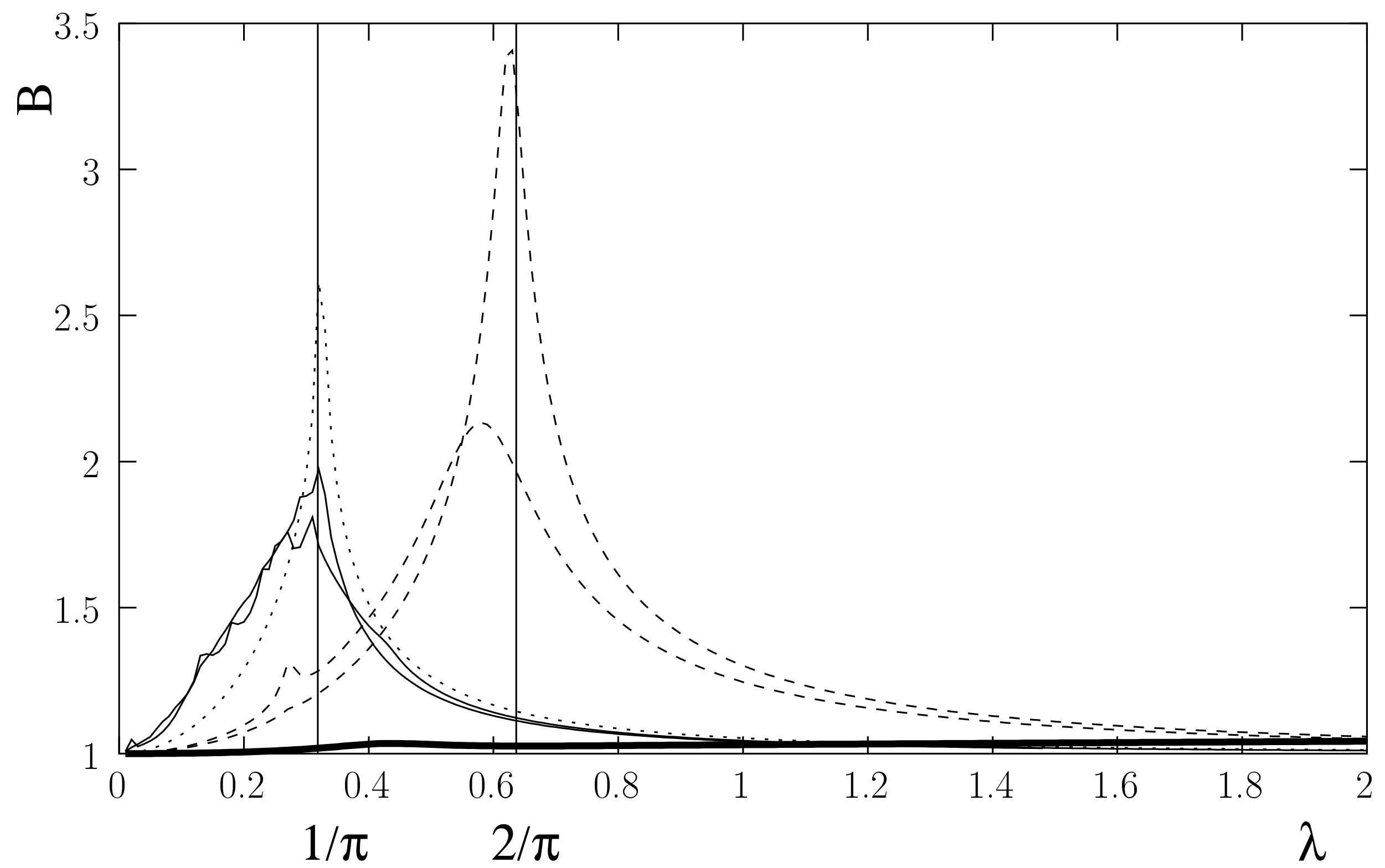

Fig. 3 


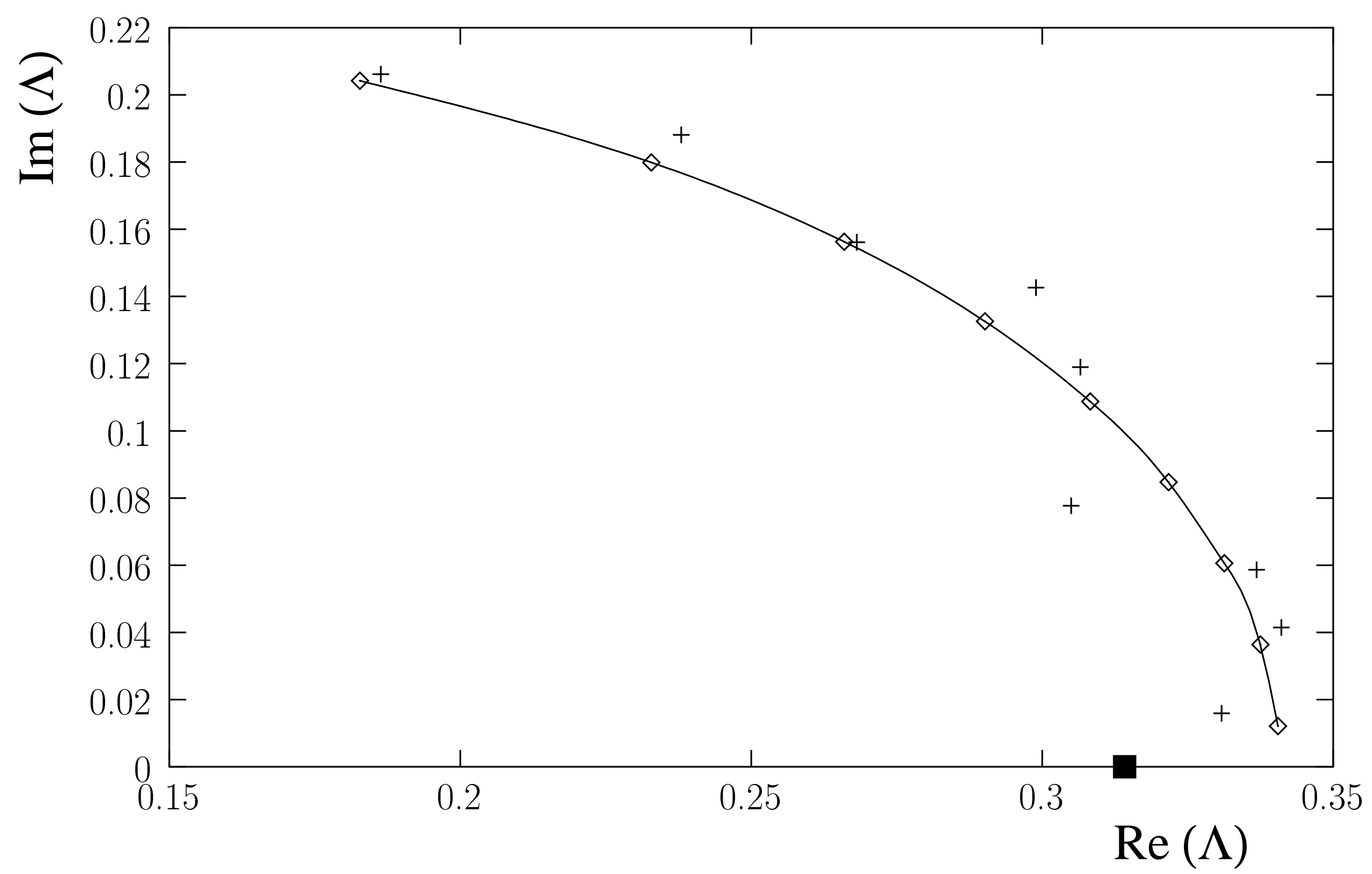

Fig. 4 